\title{
Executive Dysfunction and Behavioral Symptoms Are Associated with Deficits in Instrumental Activities of Daily Living in Frontotemporal Dementia
}

\author{
Negar Moheb ${ }^{a} \quad$ Mario F. Mendez ${ }^{a-c}$ Sarah A. Kremen ${ }^{a} \quad$ Edmond Tenga, \\ Departments of a Neurology and b Psychiatry and Behavioral Sciences, David Geffen \\ School of Medicine at University of California, Los Angeles, and ' Veterans Affairs Greater \\ Los Angeles Healthcare System, Los Angeles, CA, USA
}

\section{Keywords}

Frontotemporal lobar degeneration - Primary progressive aphasia - Semantic dementia .

Progressive nonfluent aphasia $\cdot$ Cognition $\cdot$ Behavioral symptoms

\begin{abstract}
Background: Deficits in instrumental activities of daily living (ADLs) may be more prominent in behavioral variant frontotemporal dementia (bvFTD) than in nonfluent/agrammatic variant primary progressive aphasia (nfVPPA) or semantic variant primary progressive aphasia (sVPPA). It is uncertain whether frontotemporal dementia (FTD) subgroups exhibit different patterns and/or predictors of functional impairment. Methods: We examined data from participants diagnosed with bvFTD $(n=607)$, svPPA $(n=132)$, and nfvPPA $(n=155)$ who were included in the National Alzheimer's Coordinating Center (NACC) Uniform Data Set (UDS) and assessed with the Functional Activities Questionnaire (FAQ). Stepwise multiple linear regression analyses were performed to identify associations between FAQ scores and cognitive/behavioral deficits using the NACC UDS neuropsychological testing battery and the Neuropsychiatric Inventory Questionnaire. Results: FAQ scores were higher in bvFTD than SVPPA or nfvPPA. Functional deficits across FTD subtypes differed in severity, but not pattern, and were driven by executive dysfunction and behavioral symptoms. Conclusion: Executive dysfunction and behavioral symptoms underlie instrumental ADL deficits in FTD, which are most prominent in bvFTD.


Dementia

Cognitive Disorders

\begin{tabular}{l|l}
\hline \multicolumn{2}{l}{ Dement Geriatr Cogn Disord 2017;43:89-99 } \\
\hline DOI: $10.1159 / 000455119$ & $\begin{array}{l}\text { C 2017 S. Karger AG, Basel } \\
\text { www.karger.com/dem }\end{array}$ \\
\hline
\end{tabular}

Moheb et al.: Executive Dysfunction and Behavioral Symptoms Are Associated with Deficits in Instrumental Activities of Daily Living in Frontotemporal Dementia

\section{Introduction}

Frontotemporal dementia (FTD) syndromes are a common cause of young-onset dementia in individuals under 65 years of age [1]. FTD encompasses a heterogeneous spectrum of neurodegenerative syndromes that produce early declines in social behavior, executive functioning, and/or language. Clinically, FTD has been classified into 3 subtypes: (1) behavioral variant FTD (bvFTD), which is characterized by personality changes such as behavioral disinhibition, apathy, repetitive behaviors, and loss of sympathy/empathy; (2) semantic variant primary progressive aphasia (svPPA), which is characterized by loss of semantic knowledge with intact speech fluency; and (3) nonfluent/agrammatic variant primary progressive aphasia (nfvPPA), which is characterized by agrammatism and expressive aphasia [1].

Despite the significant functional deficits seen in such patients, which have been associated with higher levels of caregiver distress [2], the literature examining activities of daily living (ADLs) in FTD patients is relatively sparse and limited by smaller sample sizes [3]. Most of the prior work has focused on bvFTD, which in some [4, 5] but not all [6-9] studies produced more profound functional deficits than Alzheimer disease (AD). A subset of reports have also included svPPA and/or nfvPPA [4, 5, 7, 10-12] and generally concluded that ADL performance, particularly for basic ADLs, is also poorer in bvFTD than in the other clinical FTD subtypes. However, due to the relatively smaller sizes of FTD cohorts at individual research centers, it remains uncertain whether these clinical FTD subtypes differ in their patterns of functional impairment as well as in their overall severity $[4,7]$.

It is also uncertain whether the cognitive and behavioral predictors of poorer ADLs across clinical FTD subtypes are similar to those seen across a broader range of elderly cohorts, both with and without cognitive impairment. Both executive function [13-15] and memory $[16,17]$ correlate with informant-based reports of functional performance in such groups. In FTD, cognitive measures, particularly of frontal/executive function, correlate with performance-based, but not informant-based, functional measures $[4,18,19]$. When behavioral associations with instrumental ADLs were examined, aberrant motor activity and hallucinations were most strongly associated with functional decline in $\mathrm{AD}[20,21]$, whereas apathy was the strongest predictor of functional decline in bvFTD and svPPA [11].

We examined the large cohort of participants with FTD included in the multicenter Uniform Data Set (UDS) maintained by the National Alzheimer's Coordinating Center (NACC) to determine the relative severity of instrumental ADL deficits, the pattern of specific instrumental ADL deficits, and the cognitive and behavioral correlates of such deficits among the clinical subtypes of FTD. We hypothesized that analyses of instrumental ADLs as measured by the Functional Activities Questionnaire (FAQ) [22] would confirm prior work suggesting more severe global ADL deficits in bvFTD relative to SvPPA and nfvPPA $[4,5,7,11]$. Additionally, given the broad differences in cognitive and behavioral deficits between FTD subgroups [1], we expected to find different patterns of deficits in specific FAQ categories and different cognitive and behavioral predictors for instrumental ADL deficits across these subgroups.

\section{Methods}

Research Participants

The NACC UDS includes data from 34 past and present Alzheimer Disease Centers supported by the National Institute on Aging [23, 24]. We identified 894 participants with primary clinical diagnoses of FTD and complete assessments with the FAQ (i.e., no missing values) whose data had been entered into the UDS from 2005 through the December 2015 data freeze. Participants were classified as bvFTD ( $n=607)$, svPPA $(n=132)$, and nfvPPA $(n=155)$ using the Neary [25] and Mesulam [26, 27] criteria. UDS participants diag- 
Table 1. Demographic data

\begin{tabular}{lcccc}
\hline & bvFTD & svPPA & nfvPPA & $F / \chi^{2}$ \\
\hline$n$ & 607 & 132 & 155 & \\
Age, years & $63.8(9.5)$ & $66.0(7.5)^{\mathrm{a}}$ & $68.7(8.7)^{\mathrm{a}, \mathrm{b}}$ & $18.34^{*}$ \\
Education, years & $15.0(3.1)$ & $15.1(3.0)$ & $15.4(2.6)$ & 0.51 \\
Symptom duration, years & $6.0(4.4)$ & $5.5(3.0)$ & $4.7(2.2)^{\mathrm{a}}$ & $7.07^{*}$ \\
Non-Hispanic White & $90.3 \%$ & $92.4 \%$ & $93.5 \%$ & 1.94 \\
Male & $60.5 \%$ & $51.5 \%$ & $45.2 \% \%^{\mathrm{a}}$ & $13.32^{*}$ \\
\hline
\end{tabular}

Parentheses denote standard deviation. bvFTD, behavioral variant frontotemporal dementia; nfvPPA, nonfluent/agrammatic variant primary progressive aphasia; svPPA, semantic variant primary progressive aphasia. ${ }^{*} p<0.05$; Bonferroni-corrected post hoc analyses: ${ }^{\mathrm{a}} p<0.017$ vs. bvFTD, ${ }^{\mathrm{b}} \mathrm{p}<0.017$ vs. svPPA. Scattered data were missing for education (bvFTD, 19; svPPA, 2; nfvPPA, 6) and symptom duration (bvFTD, 2; nfvPPA, 4).

nosed with primary progressive aphasia who did not meet the criteria for svPPA or nfvPPA (i.e., logopenic progressive aphasia), corticobasal syndrome, progressive supranuclear palsy, or mixed presentations of FTD were not included in our analyses. Written informed consent, approved by the institutional review board of each Alzheimer Disease Center, was obtained from each participant and/or their authorized representative.

\section{Functional Assessment}

The FAQ is administered to informants, who rate each participant's performance over the preceding 4 weeks across 10 separate instrumental ADL categories: (1) writing checks, paying bills, keeping financial records; (2) assembling tax or business records; (3) shopping alone; (4) playing a game of skill; (5) making coffee or tea; (6) preparing a balanced meal; (7) keeping track of current events; (8) attending to and understanding a television program, book, or magazine; (9) remembering appointments, family occasions, and medications; and (10) traveling out of the neighborhood [22]. Each category is rated on a scale between 0 and 3; higher scores denote increasing impairment. Overall FAQ performance was evaluated using total FAQ scores.

\section{Cognitive and Behavioral Assessments}

The UDS includes a standardized neuropsychological battery comprised of Trail-Making Test Parts A and B [28], the Logical Memory IA and IIA subtests from the revised Wechsler Memory Scale [29], categorical verbal fluency (animals and vegetables) [30], the forward and reverse digit span subtests from the revised Wechsler Memory Scale [29], the digit symbol subtest from the revised Wechsler Adult Intelligence Scale [31], and the odd-numbered items on the Boston Naming Test [32]. The Neuropsychiatric Inventory Questionnaire (NPI-Q) [33] is an informant-rated scale that assesses the severity (but not frequency) of 12 categories of behavioral symptoms - (1) delusions, (2) hallucinations, (3) agitation, (4) depression, (5) anxiety, (6) elation, (7) apathy, (8) disinhibition, (9) irritability, (10) aberrant motor behavior, (11) nighttime behaviors, and (12) appetitive behaviors - on a scale from 0 to 3; higher scores indicate greater severity. Overall behavioral impairment was evaluated using total NPI-Q scores across all categories.

\section{Statistical Analyses}

Statistical analyses were performed using SPSS 23 for Mac (IBM, Armonk, NY, USA). Demographic variables were compared between FTD subtypes using analysis of variance (ANOVA; continuous variables) and Kruskal-Wallis tests (categorical variables). Analysis of covariance (ANCOVA) adjusted for demographic factors was used to compare FAQ indices between FTD subgroups. Stepwise multiple regression analysis was used to determine the relative contributions of cognitive and behavioral symptomatology to functional deficits measured with the FAQ. Z-scores for separate cognitive domains identified in a prior factor analysis of the NACC UDS neuropsychological battery [34] were calculated within each group for regression analyses. Post hoc analyses underwent Bonferroni correction for multiple comparisons where appropriate. 


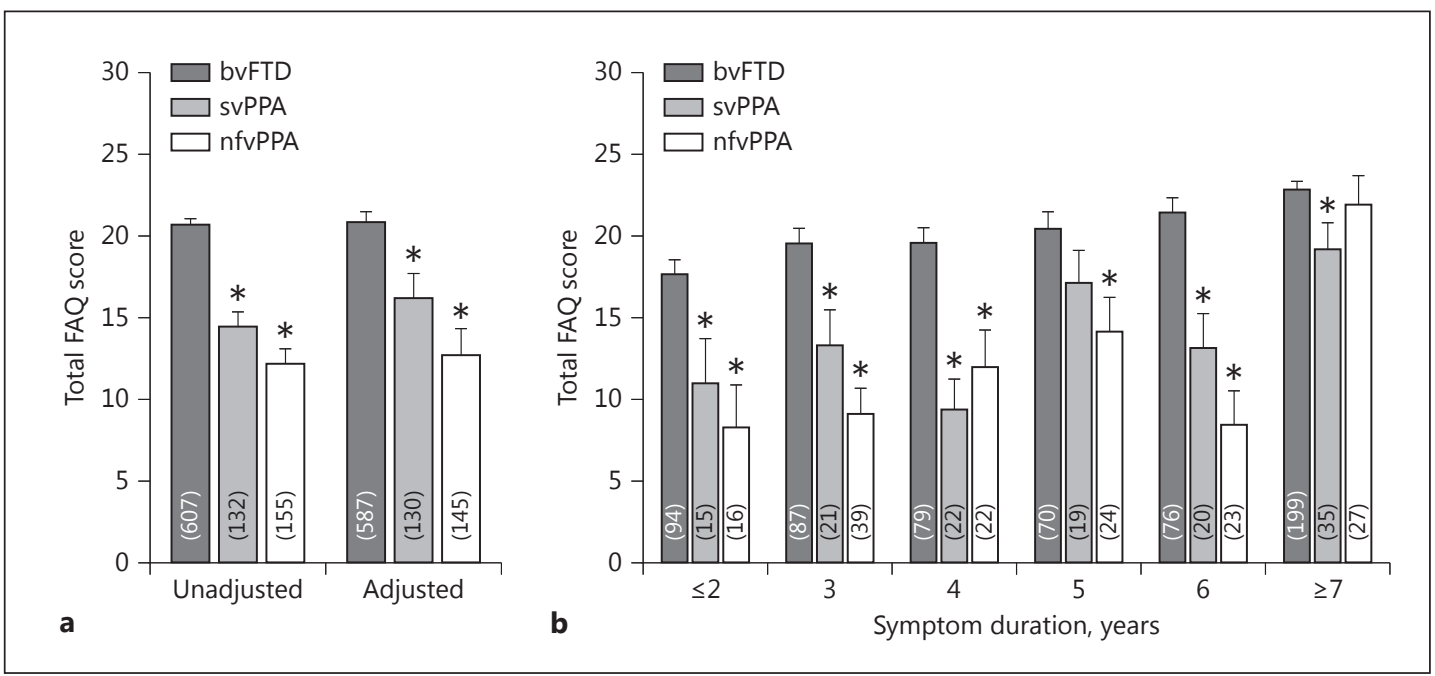

Fig. 1. Total FAQ scores across all participants in the bvFTD, svPPA, and nfvPPA groups (a) and total FAQ scores stratified by symptom duration (b). Error bars represent standard error of the mean. Parentheses denote the number of participants in each analysis. Bonferroni-corrected post hoc analyses: ${ }^{*} p<0.017$ versus bvFTD in unadjusted analyses.

\section{Results}

\section{Demographics}

The demographic data for the clinical FTD subtypes are shown in Table 1. Significant differences were seen across subtypes in age, symptom duration, and gender distribution (all $p<0.05)$. Bonferroni-corrected post hoc analyses indicated that bvFTD participants were younger than svPPA $(p=0.015)$ and nfvPPA $(p<0.001)$ participants (critical $p<0.017)$. They also had longer symptom duration at study entry $(p=0.001)$ and were more likely to be male ( $p=0.003$ ) than the nfvPPA participants. The 3 groups had similar levels of formal education and comparable ethnic distributions. Among the informants, $73.3 \%$ were spouses or partners, $15.1 \%$ were adult children, $5.0 \%$ were siblings, and $6.6 \%$ had other relationships. Informant types were similar across FTD subtypes (data not shown; $\chi_{6,894}^{2}=7.20, p=0.30$ ).

\section{FAQ Analyses}

Unadjusted and adjusted total FAQ scores for the FTD subtypes are shown in Figure 1a. One-way ANOVA indicated significant group differences on this measure $\left(F_{2,891}=70.34, p<\right.$ 0.001). Bonferroni-corrected post hoc comparisons revealed that total FAQ scores were significantly higher in the bvFTD group relative to both the svPPA and nfvPPA groups (both $p<0.001$ ), and that there was a trend towards higher FAQ scores in the svPPA group relative to the nfvPPA group ( $p=0.031$; critical $p<0.017$ ). This group effect persisted after adjustment for age, education, symptom duration, gender, and ethnicity via ANCOVA $\left(F_{2,847}=13.49, p<\right.$ $0.001)$. Post hoc group comparisons of adjusted analyses showed higher total FAQ scores in bvFTD relative to both svPPA $(p=0.015)$ and nfvPPA $(p<0.001)$, but similar scores in svPPA and nfvPPA ( $p=0.11$; critical $p<0.017)$.

Comparison of disease severity across clinical FTD subtypes remains challenging, and symptom duration represents an imperfect proxy [35]. Figure 1b shows total FAQ scores relative to symptom duration. One-way ANOVAs revealed significant group differences across symptom duration bins (all $p<0.05$ ), but the magnitude of this effect attenuated after 6 years. 


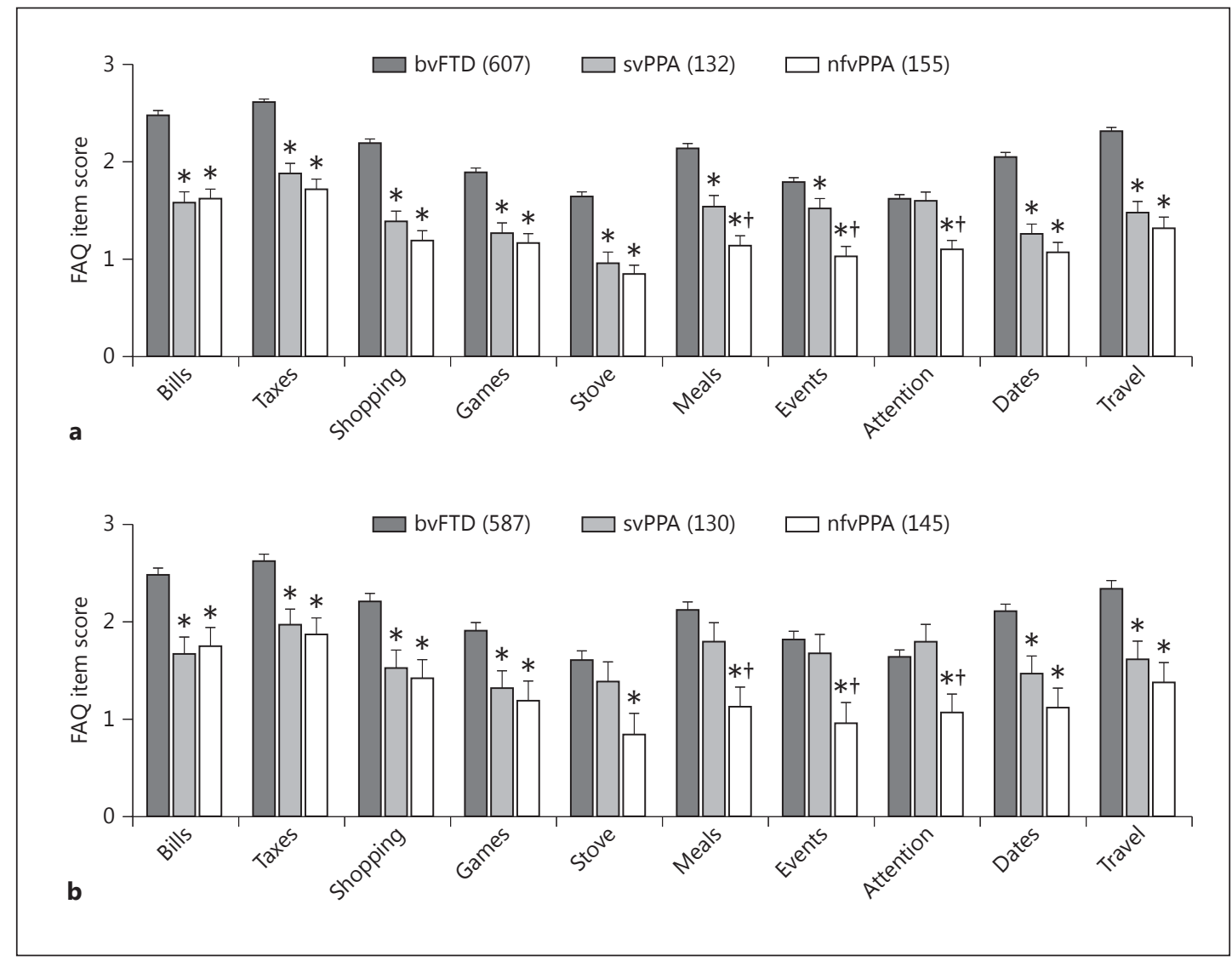

Fig. 2. Unadjusted (a) and adjusted (b) individual FAQ item scores for participants in the bvFTD, svPPA, and nfvPPA groups. Error bars represent standard error of the mean. Bonferroni-corrected post hoc analyses: $* p<0.017$ versus bvFTD and ${ }^{\dagger} p<0.017$ versus svPPA.

Since fewer participants were in each symptom duration bin, these findings were less robust to adjustment for demographic factors (data not shown).

Prior work has suggested potential qualitative differences in the pattern of functional deficits across clinical FTD subtypes [4]. We explored this question by analyzing scores from individual FAQ items (Fig. 2). Unadjusted analyses using 1-way ANOVA indicated significant group effects for all items (Fig. 2a; all $p<0.001$ ). Post hoc comparisons revealed that participants diagnosed with bvFTD had significantly higher scores on all individual FAQ items relative to those with nfvPPA (all $p<0.001$ ) and on all items other than the attention item relative to those with svPPA (all $p<0.001$ ). The svPPA group had significantly higher scores than the nfvPPA group on the events and attention items (both $p \leq 0.001$ ). After adjusting for demographic factors and symptom duration, significant group effects remained for each individual item (Fig. $2 \mathrm{~b}$; all $p<0.01$ ), with scores in the bvFTD group consistently higher than those in the nfvPPA group (all $p \leq 0.001$ ). However, a more mixed picture was seen with adjusted item scores in the svPPA group, which performed similarly to the bvFTD group on some items and similarly to the nfvPPA group on others.

\section{NPI-Q and Neuropsychological Indices}

Total NPI-Q severity scores and individual neuropsychological test scores for the FTD subgroups are shown in Table 2. Significant group effects were seen with unadjusted analyses 
Table 2. NPI-Q and neuropsychological test scores in the bvFTD, svPPA, and nfvPPA groups

\begin{tabular}{|c|c|c|c|c|c|c|c|c|}
\hline & \multicolumn{2}{|c|}{ bvFTD } & \multicolumn{2}{|c|}{ svPPA } & \multicolumn{2}{|c|}{ nfvPPA } & \multicolumn{2}{|l|}{$F$} \\
\hline & $n$ & score & $n$ & score & $n$ & score & unadjusted & adjusted \\
\hline Total NPI-Q & 595 & $10.6(6.0)$ & 130 & $7.5^{\mathrm{a}, \mathrm{c}}(5.6)$ & 151 & $5.3^{\mathrm{a}, \mathrm{b}, \mathrm{c}, \mathrm{d}}(5.4)$ & $55.08^{*}$ & $9.48^{*}$ \\
\hline Logical Memory IA & 425 & $6.3(4.8)$ & 77 & $3.5^{\mathrm{a}}(3.6)$ & 100 & $6.1^{\mathrm{b}}(5.1)$ & $11.00 *$ & 2.05 \\
\hline Logical Memory IIA & 417 & $4.7(4.6)$ & 76 & $2.4^{\mathrm{a}}(3.4)$ & 96 & $5.6^{\mathrm{b}}(4.8)$ & $11.03^{*}$ & $3.47^{*}$ \\
\hline Forward digit span & 457 & $5.6(1.5)$ & 92 & $5.5(1.8)$ & 112 & $4.5^{\mathrm{a}, \mathrm{b}, \mathrm{c}, \mathrm{d}}(1.7)$ & $18.86^{*}$ & $3.61^{*}$ \\
\hline Reverse digit span & 450 & $3.3(1.5)$ & 91 & $3.7(1.6)$ & 108 & $2.8^{\mathrm{a}, \mathrm{b}}(1.3)$ & 8.88* & 2.87 \\
\hline Animals & 462 & $9.8(6.2)$ & 96 & $6.1^{\mathrm{a}}(4.4)$ & 111 & $9.8^{b}(6.0)$ & $15.97^{*}$ & 1.95 \\
\hline Vegetables & 430 & $6.2(4.2)$ & 74 & $3.7^{\mathrm{a}, \mathrm{c}}(3.6)$ & 100 & $7.1^{\mathrm{b}, \mathrm{d}}(4.7)$ & $14.18^{*}$ & $4.07^{*}$ \\
\hline Boston Naming Test & 433 & $20.5(8.3)$ & 78 & $5.9^{\mathrm{a}, \mathrm{c}}(5.9)$ & 103 & $18.8^{\mathrm{b}, \mathrm{d}}(9.4)$ & $102.71^{*}$ & $19.06^{*}$ \\
\hline Trail-Making Test A & 392 & $66.4(40.5)$ & 84 & $55.2(39.1)$ & 101 & $68.4(42.5)$ & $3.02 *$ & 0.35 \\
\hline Trail-Making Test B & 296 & $180.2(94.3)$ & 74 & $144.8^{\mathrm{a}}(90.6)$ & 90 & $195.8^{\mathrm{b}}(87.2)$ & $6.52^{*}$ & 0.36 \\
\hline Digit symbol & 380 & $30.1(16.4)$ & 80 & $40.3^{\mathrm{a}}(17.2)$ & 100 & $28.8^{\mathrm{b}}(16.0)$ & $14.37^{*}$ & 1.83 \\
\hline
\end{tabular}

Parentheses denote standard deviation. bvFTD, behavioral variant frontotemporal dementia; nfvPPA, nonfluent/agrammatic variant primary progressive aphasia; svPPA, semantic variant primary progressive aphasia. ${ }^{*} p<0.05$. Bonferronicorrected post hoc analyses: unadjusted: ${ }^{\mathrm{a}} p<0.017$ vs. bvFTD, ${ }^{\mathrm{b}} p<0.017$ vs. svPPA; adjusted: ${ }^{\mathrm{c}} p<0.017$ vs. bvFTD, ${ }^{\mathrm{d}} \mathrm{p}<0.017$ vs. svPPA.

Table 3. Multiple linear regression analyses of total FAQ scores versus demographics, behavioral symptoms, and neuropsychological performance for the bvFTD, svPPA, and nfvPPA groups

\begin{tabular}{|c|c|c|c|c|c|c|c|c|c|}
\hline & \multicolumn{3}{|c|}{ bvFTD $(n=264)$} & \multicolumn{3}{|c|}{ svPPA $(n=57)$} & \multicolumn{3}{|c|}{ nfvPPA $(n=66)$} \\
\hline & $\beta$ & $t$ & $p$ & $\beta$ & $t$ & $p$ & $\beta$ & $t$ & $p$ \\
\hline Age & 0.07 & 1.28 & 0.200 & 0.06 & 0.74 & 0.461 & -0.03 & -0.44 & 0.657 \\
\hline Gender & 0.00 & 0.02 & 0.980 & -0.01 & -0.11 & 0.906 & 0.16 & 1.89 & 0.063 \\
\hline Education & 0.08 & 0.53 & 0.594 & -0.11 & -1.27 & 0.210 & 0.06 & 0.82 & 0.411 \\
\hline Ethnicity & 0.02 & 0.47 & 0.636 & -0.05 & -0.54 & 0.588 & 0.04 & 0.54 & 0.587 \\
\hline NPI total score & 0.34 & 6.18 & $<0.001$ & 0.58 & 6.36 & $<0.001$ & 0.24 & 3.06 & 0.003 \\
\hline Executive z & -0.24 & -4.20 & $<0.001$ & -0.52 & -5.17 & $<0.001$ & -0.51 & -5.40 & $<0.001$ \\
\hline Memory z & -0.19 & -3.29 & 0.001 & - & - & - & -0.38 & -4.42 & $<0.001$ \\
\hline Overall model & \multicolumn{3}{|c|}{$r=0.483$} & \multicolumn{3}{|c|}{$r=0.788$} & \multicolumn{3}{|c|}{$r=0.810$} \\
\hline
\end{tabular}

Reference conditions: gender: male; ethnicity: non-Hispanic White. $\beta$, standardized $\beta$ estimate; bvFTD, behavioral variant frontotemporal dementia; nfvPPA, nonfluent/agrammatic variant primary progressive aphasia; svPPA, semantic variant primary progressive aphasia.

on the NPI-Q and all neuropsychological measures. These effects persisted after adjustment for demographic factors and symptom duration on the total NPI-Q severity score, Logical Memory IIA, forward digit span, category fluency for vegetables, and the odd-numbered items on the Boston Naming Test (all $p<0.05$ ).

\section{Correlations between FAQ, NPI-Q, and Neuropsychological Indices}

A subset of the overall study cohort had complete data for demographic, FAQ, NPI-Q, and neuropsychological testing indices. Using multiple linear regression analyses, we investigated the association between these variables in each diagnostic group (Table 3). Demo- 
Table 4. Multiple linear regression analyses of total FAQ scores versus demographics and individual NPI-Q symptoms for the bvFTD, svPPA, and nfvPPA groups

\begin{tabular}{|c|c|c|c|c|c|c|c|c|c|}
\hline & \multicolumn{3}{|c|}{ bvFTD $(n=576)$} & \multicolumn{3}{|c|}{$\operatorname{svPPA}(n=128)$} & \multicolumn{3}{|c|}{ nfvPPA $(n=145)$} \\
\hline & $\beta$ & $t$ & $p$ & $\beta$ & $t$ & $p$ & $\beta$ & $t$ & $p$ \\
\hline Age & 0.06 & 1.62 & 0.104 & 0.14 & 1.76 & 0.080 & 0.12 & 1.60 & 0.112 \\
\hline Gender & 0.71 & 1.04 & 0.296 & 0.01 & 0.18 & 0.852 & -0.12 & -1.57 & 0.118 \\
\hline Education & -0.16 & -1.45 & 0.147 & -0.12 & -1.44 & 0.151 & -0.03 & -0.41 & 0.677 \\
\hline Ethnicity & -0.86 & -0.74 & 0.457 & -0.21 & -2.72 & 0.007 & 0.02 & 0.38 & 0.703 \\
\hline Hallucinations & 0.11 & 2.80 & 0.005 & - & - & - & - & - & - \\
\hline Delusions & - & - & - & - & - & - & 0.15 & 1.99 & 0.048 \\
\hline Depression & -0.09 & -2.24 & 0.025 & - & - & - & - & - & - \\
\hline Elation & -0.11 & -2.63 & 0.009 & - & - & - & - & - & - \\
\hline Apathy & 0.09 & 2.18 & 0.030 & 0.33 & 3.74 & $<0.001$ & - & - & - \\
\hline Anxiety & 0.11 & 2.66 & 0.008 & - & - & - & - & - & - \\
\hline Appetite/eating & - & - & - & - & - & - & 0.22 & 2.70 & 0.008 \\
\hline Motor behavior & 0.17 & 3.89 & $<0.001$ & 0.18 & 2.05 & 0.042 & 0.24 & 2.96 & 0.004 \\
\hline Overall model & \multicolumn{3}{|c|}{$r=0.324$} & \multicolumn{3}{|c|}{$r=0.505$} & \multicolumn{3}{|c|}{$r=0.464$} \\
\hline
\end{tabular}

Reference conditions: gender: male; ethnicity: non-Hispanic White. $\beta$, standardized $\beta$ estimate; bvFTD, behavioral variant frontotemporal dementia; nfvPPA, nonfluent/agrammatic variant primary progressive aphasia; svPPA, semantic variant primary progressive aphasia.

graphic variables were forced into each analysis, while behavioral and cognitive variables were entered in a stepwise fashion. In all 3 subtypes of FTD, total FAQ scores significantly correlated with NPI-Q severity and the processing speed/executive z-score. In bvFTD and nfvPPA, total FAQ scores also significantly correlated with the memory z-score. However, the language and attention z-scores did not correlate with FAQ scores in any of the 3 groups.

\section{Correlations between FAQ and Specific NPI-Q Symptoms}

We performed additional multiple regression analyses in the larger subset of participants with complete demographic and NPI-Q data to determine which individual behavioral symptom categories were associated with functional abilities in each clinical subgroup (Table 4). Demographic variables were forced into each analysis, while individual NPI-Q items were entered in a stepwise fashion. In bvFTD, total FAQ scores were positively correlated with the severity of hallucinations, anxiety, apathy, and aberrant motor behavior, and negatively correlated with the severity of depression and elation. In SVPPA, total FAQ scores were positively correlated with the severity of apathy and aberrant motor behavior. In nfvPPA, total FAQ scores were positively correlated with the severity of delusions, appetitive disturbances, and aberrant motor behavior.

\section{Discussion}

Our analyses of FAQ indices in a large cohort of FTD participants included in the NACC UDS confirm earlier work from smaller samples indicating more severe functional impairment in bvFTD relative to svPPA and nfvPPA $[4,5,7,11]$. After adjustment for group differences in age, gender, ethnicity, education, and symptom duration, bvFTD participants had the greatest deficits in instrumental ADLs, even at earlier stages of disease progression. 
Moheb et al.: Executive Dysfunction and Behavioral Symptoms Are Associated with Deficits in Instrumental Activities of Daily Living in Frontotemporal Dementia

There were no specific differences in patterns of impairment on the FAQ between FTD subgroups in item-level analyses. However, decreased instrumental ADL performance corresponded to poorer processing speed/executive functioning and more severe behavioral symptoms, which are characteristic of bvFTD [1]. Furthermore, poorer instrumental ADLs were not associated with greater language impairment, even in the svPPA and nfvPPA subgroups.

Despite the larger sample size available through the NACC UDS, we did not identify distinct patterns of deficits on specific FAQ items between clinical FTD subtypes. These findings suggest that the functional impairments that emerge in FTD involve a wide spectrum of everyday activities, and that differences between clinical subtypes are primarily quantitative rather than qualitative in nature. Mioshi et al. [4] had previously suggested different patterns of ADL impairment between FTD subtypes. However, the differences reported in their study were primarily driven by group differences in basic ADLs, since all 3 subtypes had similar levels of impairment on instrumental ADLs.

While clinical subtypes of FTD have similar overall patterns of functional impairment, such deficits may be associated with slightly different underlying patterns of cognitive impairment. In all 3 FTD subtypes, higher total FAQ scores were associated with poorer performance on processing speed/executive function measures. However, poorer instrumental ADL performance was associated with memory impairment only in the bvFTD and nfvPPA groups. These results are largely consistent with earlier work from broader cohorts of both cognitively intact and impaired individuals which suggests that both executive [13$15]$ and memory $[16,17]$ function contribute significantly to functional ability. Although prior studies of functional impairment in bvFTD have shown that frontal/executive or global cognitive indices correlate with performance-based ADL assessments $[18,19]$, they have largely failed to demonstrate similar associations with informant-rated ADL assessments that are more similar to the FAQ $[4,18,19]$, possibly because of their smaller sample sizes. Somewhat surprisingly, performance on language assessments did not significantly correlate with FAQ scores in either the svPPA or the nfvPPA groups. Language function may have relatively less impact on the instrumental ADLs measured by the FAQ. Alternatively, the NACC UDS neuropsychological assessments may not be sufficiently comprehensive to fully capture the language deficits that characterize these conditions.

For all 3 clinical FTD subtypes, more severe overall behavioral pathology (as measured by total NPI-Q scores) was associated with poorer instrumental ADLs. While these results are concordant with prior work suggesting associations between functional impairment and neuropsychiatric symptoms in both $\mathrm{AD}$ and vascular dementia $[20,36,37]$, they were not seen in a prior smaller study of behavioral symptoms and functional performance in bvFTD [9]. Nevertheless, targeted analyses focusing on specific neuropsychiatric symptoms have suggested that apathy is associated with poorer ADLs in bvFTD [38], similar to prior reports from $\mathrm{AD}$ and vascular dementia cohorts [20,36,37], and more pronounced longitudinal declines in instrumental ADLs in bvFTD and svPPA [11]. Likewise, we found significant correlations between apathy severity on the NPI-Q and total FAQ scores in both the bvFTD and svPPA groups, but not in the nfvPPA group. Indeed, the only NPI-Q symptom that was consistently associated with functional impairment across FTD subtypes was aberrant motor behavior, which also correlates with poorer instrumental ADLs in AD [20, 21].

Overall, the neuropsychiatric predictors for total FAQ scores in our analyses were more diverse than the cognitive predictors. Different sets of NPI-Q symptoms were positively correlated with poorer functional abilities in bvFTD (hallucinations and anxiety) versus nfvPPA (delusions and appetitive/eating disorders). Conversely, the severity of depression and elation were inversely correlated with the severity of functional impairment in bvFTD. Unlike apathy and aberrant motor activity, which progressively increase with worsening bvFTD 
[39], depression and elation remain stable or even decrease with disease progression [39, $40]$. These mood symptoms may be more prominent at milder stages of bvFTD, when functional impairments are less severe.

The large cohort of FTD participants included in the NACC UDS was a key strength of this study and allowed for more detailed analyses and comparisons of instrumental ADLs in clinical subtypes of FTD than in prior reports $[4,5,7,11]$. However, a number of factors may limit the interpretation of our results. The NACC UDS is not specifically designed for analyses of FTD, and some of the standardized assessments may fail to capture the more unique deficits seen in this spectrum of disorders. In particular, it is difficult to compare dementia severity across FTD subtypes, given their very different clinical presentations. We chose to use symptom duration as a potential surrogate; others have used the global Clinical Dementia Rating (CDR) [41] for this purpose [39, 40]. The standard CDR was less well suited for our analyses, given its focus on AD symptomatology and inclusion of functional decline in its scoring. Other measures such as the frontotemporal lobar degeneration-modified CDR [42, 43] or the Frontotemporal Dementia Rating Scale [35] appear to be more effective in staging severity across different types of dementia. Future analyses using such instruments may provide further insight into the questions addressed in this study. Our analyses were based upon clinical diagnoses of FTD using older criteria [25-27] that have since been supplanted by newer criteria which may be more sensitive [44-46] and identify patients at earlier stages of disease that are characterized by less functional impairment. Diagnoses in this study lack neuropathological confirmation. Since a prior study of NACC participants with clinical diagnoses of bvFTD revealed that a significant proportion had AD pathology [47] and since differences in the clinical symptoms of FTD appear to be driven in part by differences in underlying neuropathology $[10,47,48]$, further analyses of ADLs in FTD should incorporate clinicopathological correlations.

Overall, our findings confirm and extend prior reports that ADLs, including instrumental ADLs, are more impaired in bvFTD than in svPPA and nfvPPA. Across FTD subgroups, poorer function was most consistently associated with executive dysfunction and behavioral symptoms, but relatively independent of language deficits. Taken together, these results raise the possibility that interventions tailored at behavioral pathology across the clinical subtypes of FTD may have the potential to improve functional outcomes.

\section{Acknowledgments}

This research was supported by the Alzheimer's Disease Research Centers of California and the Sidell-Kagan Foundation. The NACC database is funded by NIA/NIH grant U01 AG016976. NACC data were contributed by the NIA-funded Alzheimer Disease Centers: P30 AG019610 (PI Eric Reiman, MD), P30 AG013846 (PI Neil Kowall, MD), P50 AG008702 (PI Scott Small, MD), P50 AG025688 (PI Allan Levey, MD, PhD), P50 AG047266 (PI Todd Golde, MD, PhD), P30 AG010133 (PI Andrew Saykin, PsyD), P50 AG005146 (PI Marilyn Albert, PhD), P50 AG005134 (PI Bradley Hyman, MD, PhD), P50 AG016574 (PI Ronald Petersen, MD, PhD), P50 AG005138 (PI Mary Sano, PhD), P30 AG008051 (PI Steven Ferris, PhD), P30 AG013854 (PI M. Marsel Mesulam, MD), P30 AG008017 (PI Jeffrey Kaye, MD), P30 AG010161 (PI David Bennett, MD), P50 AG047366 (PI Victor Henderson, MD, MS), P30 AG010129 (PI Charles DeCarli, MD), P50 AG016573 (PI Frank LaFerla, PhD), P50 AG016570 (PI Marie-Francoise Chesselet, MD, PhD), P50 AG005131 (PI Douglas Galasko, MD), P50 AG023501 (PI Bruce Miller, MD), P30 AG035982 (PI Russell Swerdlow, MD), P30 AG028383 (PI Linda Van Eldik, PhD), P30 AG010124 (PI John Trojanowski, MD, PhD), P50 AG005133 (PI Oscar Lopez, MD), P50 AG005142 (PI Helena Chui, MD), P30 AG012300 (PI Roger Rosenberg, MD), P50 AG005136 (PI Thomas Montine, MD, PhD), P50 AG033514 (PI Sanjay Asthana, MD, FRCP), P50 AG005681 (PI John Morris, MD), and P50 AG047270 (PI Stephen Strittmatter, MD, PhD). 
Moheb et al.: Executive Dysfunction and Behavioral Symptoms Are Associated with

Deficits in Instrumental Activities of Daily Living in Frontotemporal Dementia

\section{Disclosure Statement}

The authors have no conflicts of interest or other disclosures to report.

\section{References}

1 Bang J, Spina S, Miller BL: Frontotemporal dementia. Lancet 2015;386:1672-1682.

-2 Lima-Silva TB, Bahia VS, Carvalho VA, Guimaraes HC, Caramelli P, Balthazar ML, Damasceno B, Bottino CM, Brucki SM, Nitrini R, Yassuda MS: Neuropsychiatric symptoms, caregiver burden and distress in behavioralvariant frontotemporal dementia and Alzheimer's disease. Dement Geriatr Cogn Disord 2015;40:268-275.

- 3 Lima-Silva TB, Bahia VS, Nitrini R, Yassuda MS: Functional status in behavioral variant frontotemporal dementia: a systematic review. Biomed Res Int 2013;2013:837120.

-4 Mioshi E, Kipps CM, Dawson K, Mitchell J, Graham A, Hodges JR: Activities of daily living in frontotemporal dementia and Alzheimer disease. Neurology 2007;68:2077-2084.

5 Borroni B, Padovani A: Re: Activities of daily living in frontotemporal dementia and Alzheimer disease. Neurology 2007;69:2110.

6 Piquard A, Derouesne C, Lacomblez L, Sieroff E: Planning and activities of daily living in Alzheimer's disease and frontotemporal dementia (in French). Psychol Neuropsychiatr Vieil 2004;2:147-156.

7 Wicklund AH, Johnson N, Rademaker A, Weitner BB, Weintraub S: Profiles of decline in activities of daily living in non-Alzheimer dementia. Alzheimer Dis Assoc Disord 2007;21:8-13.

-8 Larner AJ, Hancock P: Re: Activities of daily living in frontotemporal dementia and Alzheimer disease. Neurology 2008;70:658.

-9 Boutoleau-Bretonniere C, Vercelletto M, Volteau C, Renou P, Lamy E: Zarit Burden Inventory and activities of daily living in the behavioral variant of frontotemporal dementia. Dement Geriatr Cogn Disord 2008;25:272277.

10 Mioshi E, Hodges JR: Rate of change of functional abilities in frontotemporal dementia. Dement Geriatr Cogn Disord 2009;28:419-426.

11 O'Connor CM, Clemson L, Hornberger M, Leyton CE, Hodges JR, Piguet O, Mioshi E: Longitudinal change in everyday function and behavioral symptoms in frontotemporal dementia. Neurol Clin Pract 2016;6:419-428.

$>12$ O'Connor CM, Clemson L, Flanagan E, Kaizik C, Brodaty H, Hodges JR, Piguet O, Mioshi E: The relationship between behavioural changes, cognitive symptoms, and functional disability in primary progressive aphasia: a longitudinal study. Dement Geriatr Cogn Disord 2016;42:215-226.

13 Bell-McGinty S, Podell K, Franzen M, Baird AD, Williams MJ: Standard measures of executive function in predicting instrumental activities of daily living in older adults. Int J Geriatr Psychiatry 2002;17:828-834.

-14 Cahn-Weiner DA, Boyle PA, Malloy PF: Tests of executive function predict instrumental activities of daily living in community-dwelling older individuals. Appl Neuropsychol 2002;9:187-191.

15 Pereira FS, Yassuda MS, Oliveira AM, Forlenza OV: Executive dysfunction correlates with impaired functional status in older adults with varying degrees of cognitive impairment. Int Psychogeriatr 2008;20:1104-1115.

16 Goldstein G, McCue M, Rogers J, Nussbaum PD: Diagnostic differences in memory test based predictions of functional capacity in the elderly. Neuropsychol Rehabil 1992;2:307-317.

$\checkmark 17$ Farias ST, Mungas D, Reed B, Haan MN, Jagust WJ: Everyday functioning in relation to cognitive functioning and neuroimaging in community-dwelling Hispanic and non-Hispanic older adults. J Int Neuropsychol Soc 2004;10:342-354.

18 Mioshi E, Kipps CM, Hodges JR: Activities of daily living in behavioral variant frontotemporal dementia: differences in caregiver and performance-based assessments. Alzheimer Dis Assoc Disord 2009;23:70-76.

19 Lima-Silva TB, Bahia VS, Carvalho VA, Guimaraes HC, Caramelli P, Balthazar ML, Damasceno B, Bottino CM, Brucki SM, Nitrini R, Yassuda MS: Direct and indirect assessments of activities of daily living in behavioral variant frontotemporal dementia and Alzheimer disease. J Geriatr Psychiatry Neurol 2015;28:19-26. Tekin S, Fairbanks LA, O'Connor S, Rosenberg S, Cummings JL: Activities of daily living in Alzheimer's disease: neuropsychiatric, cognitive, and medical illness influences. Am J Geriatr Psychiatry 2001;9:81-86.

21 Mok WY, Chu LW, Chung CP, Chan NY, Hui SL: The relationship between non-cognitive symptoms and functional impairment in Alzheimer's disease. Int J Geriatr Psychiatry 2004;19:1040-1046.

-22 Pfeffer RI, Kurosaki TT, Harrah CH Jr, Chance JM, Filos S: Measurement of functional activities in older adults in the community. J Gerontol 1982;37:323-329.

-23 Morris JC, Weintraub S, Chui HC, Cummings J, Decarli C, Ferris S, Foster NL, Galasko D, Graff-Radford N, Peskind ER, Beekly D, Ramos EM, Kukull WA: The Uniform Data Set (UDS): clinical and cognitive variables and descriptive data from Alzheimer Disease Centers. Alzheimer Dis Assoc Disord 2006;20:210-216.

24 Beekly DL, Ramos EM, Lee WW, Deitrich WD, Jacka ME, Wu J, Hubbard JL, Koepsell TD, Morris JC, Kukull WA: The National Alzheimer's Coordinating Center (NACC) database: the Uniform Data Set. Alzheimer Dis Assoc Disord 2007;21:249-258.

25 Neary D, Snowden JS, Gustafson L, Passant U, Stuss D, Black S, Freedman M, Kertesz A, Robert PH, Albert M, Boone K, Miller BL, Cummings J, Benson DF: Frontotemporal lobar degeneration: a consensus on clinical diagnostic criteria. Neurology 1998;51:1546-1554. 
Moheb et al.: Executive Dysfunction and Behavioral Symptoms Are Associated with

Deficits in Instrumental Activities of Daily Living in Frontotemporal Dementia

$-26$

$-27$

28

29

9

$$
1987 .
$$

30 Morris JC, Heyman A, Mohs RC, Hughes JP, van Belle G, Fillenbaum G, Mellits ED, Clark C: The Consortium to Establish a Registry for Alzheimer's Disease (CERAD). Part I. Clinical and neuropsychological assessment of Alzheimer's disease. Neurology 1989;39:1159-1165.

31 Wechsler D: Wechsler Adult Intelligence Scale-Revised Edition: Manual. San Antonio, The Psychological Corporation, 1981.

32 Kaplan E, Goodglass H, Weintraub S: Boston Naming Test. Philadelphia, Lea \& Febiger, 1983.

-33 Kaufer DI, Cummings JL, Ketchel P, Smith V, MacMillan A, Shelley T, Lopez OL, DeKosky ST: Validation of the NPI-Q, a brief clinical form of the Neuropsychiatric Inventory. J Neuropsychiatry Clin Neurosci 2000;12:233239.

-34 Teng E, Becker BW, Woo E, Cummings JL, Lu PH: Subtle deficits in instrumental activities of daily living in subtypes of mild cognitive impairment. Dement Geriatr Cogn Disord 2010;30:189-197.

-35 Mioshi E, Hsieh S, Savage S, Hornberger M, Hodges JR: Clinical staging and disease progression in frontotemporal dementia. Neurology 2010;74:1591-1597.

-36 D’Onofrio G, Sancarlo D, Panza F, Copetti M, Cascavilla L, Paris F, Seripa D, Matera MG, Solfrizzi V, Pellegrini F, Pilotto A: Neuropsychiatric symptoms and functional status in Alzheimer's disease and vascular dementia patients. Curr Alzheimer Res 2012;9:759-771.

-37 Senanarong V, Poungvarin N, Jamjumras P, Sriboonroung A, Danchaivijit C, Udomphanthuruk S, Cummings JL: Neuropsychiatric symptoms, functional impairment and executive ability in Thai patients with Alzheimer's disease. Int Psychogeriatr 2005;17:81-90.

-38 Kipps CM, Mioshi E, Hodges JR: Emotion, social functioning and activities of daily living in frontotemporal dementia. Neurocase 2009;15:182-189.

-39 Ranasinghe KG, Rankin KP, Lobach IV, Kramer JH, Sturm VE, Bettcher BM, Possin K, Christine You S, Lamarre AK, Shany-Ur T, Stephens ML, Perry DC, Lee SE, Miller ZA, Gorno-Tempini ML, Rosen HJ, Boxer A, Seeley WW, Rabinovici GD, Vossel KA, Miller BL: Cognition and neuropsychiatry in behavioral variant frontotemporal dementia by disease stage. Neurology 2016;86:600-610.

40 Diehl-Schmid J, Pohl C, Perneczky R, Forstl H, Kurz A: Behavioral disturbances in the course of frontotemporal dementia. Dement Geriatr Cogn Disord 2006;22:352-357.

-41 Morris JC: The Clinical Dementia Rating (CDR): current version and scoring rules. Neurology 1993;43:24122414.

-42 Deutsch MB, Liang LJ, Jimenez EE, Mather MJ, Mendez MF: Are we comparing frontotemporal dementia and Alzheimer disease patients with the right measures? Int Psychogeriatr 2016;28:1481-1485.

-43 Premi E, Gualeni V, Costa P, Cosseddu M, Gasparotti R, Padovani A, Borroni B: Looking for measures of disease severity in the frontotemporal dementia continuum. J Alzheimers Dis 2016;52:1227-1235.

-44 Rascovsky K, Hodges JR, Knopman D, Mendez MF, Kramer JH, Neuhaus J, van Swieten JC, Seelaar H, Dopper EG, Onyike CU, Hillis AE, Josephs KA, Boeve BF, Kertesz A, Seeley WW, Rankin KP, Johnson JK, Gorno-Tempini ML, Rosen H, Prioleau-Latham CE, Lee A, Kipps CM, Lillo P, Piguet O, Rohrer JD, Rossor MN, Warren JD, Fox NC, Galasko D, Salmon DP, Black SE, Mesulam M, Weintraub S, Dickerson BC, Diehl-Schmid J, Pasquier F, Deramecourt V, Lebert F, Pijnenburg Y, Chow TW, Manes F, Grafman J, Cappa SF, Freedman M, Grossman M, Miller BL: Sensitivity of revised diagnostic criteria for the behavioural variant of frontotemporal dementia. Brain 2011; 134:2456-2477.

-45 Gorno-Tempini ML, Hillis AE, Weintraub S, Kertesz A, Mendez M, Cappa SF, Ogar JM, Rohrer JD, Black S, Boeve BF, Manes F, Dronkers NF, Vandenberghe R, Rascovsky K, Patterson K, Miller BL, Knopman DS, Hodges JR, Mesulam MM, Grossman M: Classification of primary progressive aphasia and its variants. Neurology 2011; 76:1006-1014.

-46 Vijverberg EG, Dols A, Krudop WA, Peters A, Kerssens CJ, van Berckel BN, Wattjes MP, Barkhof F, Gossink F, Prins ND, Stek ML, Scheltens P, Pijnenburg YA: Diagnostic accuracy of the frontotemporal dementia consensus criteria in the late-onset frontal lobe syndrome. Dement Geriatr Cogn Disord 2016;41:210-219.

-47 Mendez MF, Joshi A, Tassniyom K, Teng E, Shapira JS: Clinicopathologic differences among patients with behavioral variant frontotemporal dementia. Neurology 2013;80:561-568.

-48 Leger GC, Banks SJ: Neuropsychiatric symptom profile differs based on pathology in patients with clinically diagnosed behavioral variant frontotemporal dementia. Dement Geriatr Cogn Disord 2014;37:104-112. 